\title{
PET imaging of brain macrophages using the peripheral benzodiazepine receptor in a macaque model of neuroAIDS
}

\author{
Sriram Venneti,, ${ }^{1}$ Brian J. Lopresti,, ${ }^{2}$ Guoji Wang, ${ }^{3}$ Stephanie J. Bissel,, ${ }^{4}$ Chester A. Mathis, ${ }^{2}$ \\ Carolyn C. Meltzer, ${ }^{2}$ Fernando Boada, ${ }^{2}$ Saverio Capuano III, ${ }^{5}$ Geraldine J. Kress, ${ }^{6}$ \\ Denise K. Davis, ${ }^{2}$ James Ruszkiewicz, ${ }^{2}$ lan J. Reynolds, ${ }^{6}$ Michael Murphey-Corb, ${ }^{7}$ \\ Anita M. Trichel, ${ }^{7}$ Stephen R. Wisniewski, ${ }^{8}$ and Clayton A. Wiley ${ }^{3}$

\begin{abstract}
${ }^{1}$ Department of Neurobiology, 2Department of Radiology, and ${ }^{3}$ Department of Pathology, University of Pittsburgh School of Medicine; 4Department of Infectious Diseases \& Microbiology, Graduate School of Public Health; 5Department of Obstetrics, Gynecology \& Reproductive Sciences, ${ }^{6}$ Department of Pharmacology, and 7Department of Molecular Genetics \& Biochemistry, University of Pittsburgh School of Medicine; ${ }^{8}$ Department of Epidemiology, Graduate School of Public Health, University of Pittsburgh, Pittsburgh, Pennsylvania, USA.
\end{abstract}

\begin{abstract}
HIV infection in humans and simian immunodeficiency virus (SIV) infection in macaques result in encephalitis in approximately one-quarter of infected individuals and is characterized by infiltration of the brain with infected and activated macrophages. 1-(2-chlorphenyl)- $N$-methyl- $N$-(1-methylpropyl)-3-isoquinoline-carboxamide (PK11195) is a ligand specific for the peripheral benzodiazepine receptor abundant on macrophages and is expressed in low levels in the noninfected brain. We hypothesized that positron-emission tomography (PET) with the carbon-11-labeled, R-enantiomer form of PK11195 ([11 C](R)-PK11195) could image brain macrophages and hence the development of encephalitis in vivo. $\left[{ }^{11} \mathrm{C}\right](\mathrm{R})$-PK11195 binding was assessed in the brain using PET in 11 SIV infected macaques, six of which showed increased binding in vivo. Postmortem examination of the brain in these six macaques demonstrated encephalitis, while macaques that did not show an increase in $\left[{ }^{11} \mathrm{C}\right](\mathrm{R})-\mathrm{PK} 11195$ binding did not develop SIV encephalitis. Brain tissue from SIV encephalitic macaques also showed increased $\left[{ }^{3} \mathrm{H}\right](\mathrm{R})$-PK11195 binding compared with binding in nonencephalitic macaques. Increased PK11195 binding in vivo and in postmortem brain tissue correlated with abundance of macrophages but not astrocytes. Our results suggest that PET $\left[{ }^{11} \mathrm{C}\right](\mathrm{R})$-PK11195 imaging can detect the presence of macrophages in SIV encephalitis in vivo and may be useful to predict the development of HIV encephalitis and in studies of the pathogenesis and treatment of HIV dementia.
\end{abstract}

\begin{abstract}
Introduction
Approximately one-quarter of immunosuppressed AIDS patients develop a neurodegenerative disorder clinically characterized as HIV-associated dementia complex (1-4). Early neurological symptoms appear to be reversible; however, later a progressive neurological deficit becomes fixed (5). The simian model of lentiviral infection closely resembles the human disease with a variable percentage of infected macaques developing neurological disease (6). Histopathological hallmarks of both HIV and simian immunodeficiency virus (SIV) encephalitis (HIVE or SIVE) include abundant brain tissue infiltration by macrophages, multinucleated giant cells, microglial nodules, and perivascular chronic inflammatory cells $(7,8)$. The pathogenesis of neurodegeneration is unknown. Current hypotheses implicate viral proteins and toxins derived
\end{abstract}

Nonstandard abbreviations used: $\alpha 1$-acid glycoprotein (AGP); carbon-11-labeled,

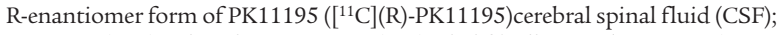
cyanine 5 (Cy5); echo-planar imaging (EPI); glial fibrillary acidic protein (GFAP); human immunodeficiency virus encephalitis (HIVE); injected dose of PK11195 per grams of brain tissue body weight of animal (ID $/ \mathrm{g} \times \mathrm{kg})$; magnetic resonance imaging (MRI); maximal bound response to ligand $\left(\mathrm{B}_{\max }\right)$; microtubule-associated protein-2 (MAP-2); peripheral benzodiazepine receptor (PBR); 1-(2-chlorophenyl)- $N$-methyl- $N$ (1-methylpropyl)-3-isoquinoline-carboxamide (PK11195); positron emission tomography (PET); simian immunodeficiency virus (SIV); simian immunodeficiency virus encephalitis (SIVE).

Conflict of interest: The authors have declared that no conflict of interest exists.

Citation for this article: $J$. Clin. Invest. 113:981-989 (2004).

doi:10.1172/JCI200420227. from both HIV-infected and activated macrophages that cause neuronal damage (9).

Several imaging modalities have been used to assess CNS changes associated with lentiviral infection (10-12). While x-ray computed tomography has been considered relatively insensitive, magnetic resonance imaging (MRI) has demonstrated a spectrum of lesions including white matter abnormalities and brain atrophy in demented HIV-infected subjects (13-18). Functional imaging studies using positron emission tomography (PET) with the fluorine-18-labeled glucose analogue $\left[{ }^{18} \mathrm{~F}\right] 2$-fluoro-2-deoxyglucose, single-photon emission computed tomography, magnetic resonance spectroscopy, and functional MRI have offered tantalizing insights into lentiviral infection, but no definitive relationship between abnormalities and onset of neurological disease has been observed (19-23). If HIVE could be evaluated during life, it would be possible to identify developing neurological damage and monitor efficacy of therapy before development of fixed deficits. Since activated and infected macrophages are the sine qua non of HIVE and SIVE $(7,9)$, it may be possible to monitor the progression of HIVE if it were possible to monitor the presence of macrophages in vivo.

Radiolabeled ligands have been recently synthesized that selectively bind to the peripheral benzodiazepine receptor (PBR), which is abundantly expressed on brain macrophages $(24,25)$. The isoquinoline carboxamide derivative 1-(2-chlorophenyl)- $N$-methyl- $N$ (1-methylpropyl)-3-isoquinoline-carboxamide (PK11195) is a spe- 
cific ligand for PBR. Because PBR is expressed only at low levels in the normal brain, several in vitro and in vivo studies have examined whether PK11195 can be used to radiolabel activated macrophages in the diseased CNS. Autoradiographic studies have demonstrated increased binding of $\left[{ }^{3} \mathrm{H}\right](\mathrm{R})$-PK11195 (where $\mathrm{R}$ indicates the R-enantiomer of PK11195) to macrophages in a wide variety of neurological diseases such as multiple sclerosis and experimental autoimmune encephalitis $(26,27)$, stroke $(28)$, and brain trauma (29). Several studies have used PET with the carbon-11-labeled, R-enantiomer form of PK11195 ( $\left.\left[{ }^{11} \mathrm{C}\right](\mathrm{R})-\mathrm{PK} 11195\right)$ to detect activated macrophages in the CNS of patients with multiple sclerosis $(26,27,30)$, Rasmussen encephalitis (31), herpes encephalitis (32), Alzheimer disease (33), and multiple system atrophy (34). In the current study we used both in vivo PET imaging and binding analysis of postmortem tissues to demonstrate increased specific binding of both $\left[{ }^{11} \mathrm{C}\right](\mathrm{R})-\mathrm{PK} 11195$ and $\left[{ }^{3} \mathrm{H}\right](\mathrm{R})$-PK11195 to macrophages in SIVE macaques. Increased PK11195-specific binding in SIVE suggests that PET using $\left[{ }^{11} \mathrm{C}\right](\mathrm{R})$-PK11195 may permit in vivo monitoring of the evolution and treatment of HIV dementia.

\section{Methods}

Animals. All animals were housed and maintained according to strict standards of the Association for Assessment and Accreditation of Laboratory Animal Care, and experiments were approved by the University of Pittsburgh Institutional Animal Care and Use Committee. Fourteen macaques: four pigtail macaques (Macaca nemestrina) and ten rhesus macaques (Macaca mulatta) varying from ages 84 to 168 months were challenged with SIVDeltaB670 (SIVDB670), with the length of infection varying from 56 to 1,622 days (Table 1).

We used six CD8 T cell-depleted macaques (to compress the disease time course) and eight nondepleted macaques in this study. Preliminary studies from our laboratory have shown that the CD8 T cell-depleted macaques develop encephalitis (unpublished data). CD8 $\mathrm{T}$ cell depletion was performed using protocols analogous to those previously described (35) using the CD8 T cell-specific Ab cM-T807 (gift from Keith Reimann, Division of Viral Pathogene- sis, Harvard Medical School, Boston, Massachusetts, USA). Eleven macaques were available for PET scanning after infection.

Macaques were sacrificed at prespecified time points or when moribund with AIDS (Table 1). Complete neuropathological microscopic analysis was performed in all macaques, and no opportunistic infections were identified in the CNS. SIVE was diagnosed and classified as mild, moderate, or severe on the basis of distribution of perivascular and parenchymal macrophage infiltrates, microglial nodules, multinucleated giant cells, and abundant macrophages that immunostained for SIV envelope protein (assessed in multiple $\times 10$ and $\times 40$ fields per brain region) in various brain regions (8) (Table 2). Brain tissue from two noninfected macaques served as additional controls.

PET imaging. High specific activity $\left[{ }^{11} \mathrm{C}\right](\mathrm{R})$-PK11195 was produced at the University of Pittsburgh Medical Center PET Facility using methods similar to those previously described by the Hammersmith PET Group (36), except that the methylation was performed at room temperature for 2 minutes to minimize the dechlorination side reaction (37). Chemical and radiochemical purities were equal to or greater than $95 \%$ with specific activities equal to or greater than $2 \mathrm{Ci} / \mu \mathrm{mol}$ at the end of a 40 -minute synthesis. The typical end-of-synthesis yields of high-purity $\left[{ }^{11} \mathrm{C}\right](\mathrm{R})$ PK11195 were in the range of 40-100 $\mathrm{mCi}$.

Chronically infected macaques were imaged using PET with $\left[{ }^{11} \mathrm{C}\right](\mathrm{R})-\mathrm{PK} 11195$ either before death (non-CD8 T cell-depleted animals) or before SIV infection and at 2 months after infection (CD8 T cell-depleted animals). After a 10-minute transmission scan using rotating ${ }^{68} \mathrm{Ga} /{ }^{68} \mathrm{Ge}$ rod sources, approximately $5 \mathrm{mCi}$ (average dose $4.92 \pm 0.6 \mathrm{mCi}$, range $3.33-5.92 \mathrm{mCi}$ ) of high specific activity $\left[{ }^{11} \mathrm{C}\right](\mathrm{R})-\mathrm{PK} 11195 \mathrm{in} 5 \mathrm{ml}$ of sterile isotonic saline was injected intravenously into each macaque. PET data were acquired in 3-D imaging mode using an ECAT $\mathrm{HR}^{+}$PET scanner (CTI PET Systems Inc., Knoxville, Tennessee, USA). This instrument simultaneously acquired 63 image planes $(15.2 \mathrm{~cm}$ field of view) with a reconstructed image resolution of $5.8 \mathrm{~mm}$ full-width half-maximum (ramp filter). A dynamic series of PET scans was acquired

\section{Table 1}

Macaque experimental parameters

\begin{tabular}{|c|c|c|c|c|c|c|c|}
\hline $\begin{array}{l}\text { Macaque } \\
\text { number }\end{array}$ & Species & $\begin{array}{l}\text { CD8 T cell } \\
\text { depletion }\end{array}$ & Virus & $\begin{array}{l}\text { Length of infection } \\
\text { (days) }\end{array}$ & $\begin{array}{l}\text { PET } \\
\text { scan }\end{array}$ & $\begin{array}{l}\text { Filtration binding analysis } \\
\text { in postmortem brain tissue }\end{array}$ & $\begin{array}{l}\text { Time of } \\
\text { sacrifice }\end{array}$ \\
\hline 1259 & Pigtailed & Yes & SIVDB670 & 66 & Yes & Yes & Moribund with AIDS \\
\hline 2263 & Rhesus & Yes & SIVDB670 & 80 & Yes & No & Moribund with AIDS \\
\hline 2265 & Rhesus & Yes & SIVDB670 & 56 & Yes & Yes & Moribund with AIDS \\
\hline 2266 & Rhesus & Yes & SIVDB670 & 56 & Yes & Yes & Moribund with AIDS \\
\hline 1220 & Pigtailed & No & SIVDB670 & 171 & Yes & No & Moribund with AIDS \\
\hline 0222 & Pigtailed & No & SIVDB670 & 121 & Yes & No & Moribund with AIDS \\
\hline 9240 & Rhesus & No & SIVDB670 & 64 & Yes & No & Timed sacrifice \\
\hline 9244 & Rhesus & No & SIVDB670 & 1622 & Yes & No & Moribund with AIDS \\
\hline 9243 & Rhesus & No & SIVDB670 & 408 & Yes & No & Moribund with AIDS \\
\hline 2229 & Rhesus & No & SIVDB670 & 722 & No & Yes & Moribund with AIDS \\
\hline 9231 & Rhesus & No & SIVDB670 & 351 & Yes & No & Timed sacrifice \\
\hline 2233 & Rhesus & No & SIVDB670 & 1062 & No & Yes & Moribund with AIDS \\
\hline 1260 & Pigtailed & Yes & SIVDB670 & 70 & Yes & No & Timed sacrifice \\
\hline 2264 & Rhesus & Yes & SIVDB670 & 69 & No & Yes & Moribund with AIDS \\
\hline 7405 & Rhesus & No & NAp & NAp & No & Yes & Not infected \\
\hline 7421 & Rhesus & No & NAp & NAp & No & Yes & Not infected \\
\hline
\end{tabular}

Time of sacrifice indicates if macaques were sacrificed at predetermined time points or sacrificed when moribund with AIDS. SIVDB670, SIVDeltaB670; NAp, not applicable. 


\section{Table 2}

Increased $\left[{ }^{11} \mathrm{C}\right](\mathrm{R})-\mathrm{PK} 11195$ binding in vivo corresponds to the presence of SIVE and increased $\left[{ }^{3} \mathrm{H}\right](\mathrm{R})$-PK11195 binding in postmortem brain tissue

\begin{tabular}{lccc}
$\begin{array}{l}\text { Macaque } \\
\text { number }\end{array}$ & $\begin{array}{c}\text { PET binding } \\
\text { value (\% increase } \\
\text { over control) }\end{array}$ & Histopathology & $\begin{array}{c}\text { Postmortem tissue } \\
\text { binding (\% increase } \\
\text { over control) }\end{array}$ \\
1259 & 313 & Severe SIVE & 104 \\
$2263^{\mathrm{A}}$ & 213 & Mild SIVEA & NA \\
2265 & 122 & Moderate SIVE & 71 \\
2266 & 174 & Severe SIVE & 37 \\
1220 & 55 & Severe SIVE & NA \\
0222 & 39 & Moderate SIVE & NA \\
9240 & 11 & No encephalitis & NA \\
9244 & 0 & No encephalitis & NA \\
9243 & 0 & No encephalitis & NA \\
2229 & NA & No encephalitis & 0 \\
$9231^{B}$ & $29 B$ & No encephalitis & NA \\
2233 & NA & No encephalitis & 9 \\
1260 & 0 & No encephalitis & NA \\
2264 & NA & No encephalitis & 1 \\
\hline
\end{tabular}

Representative data obtained from the basal ganglia of 14 SIV-infected macaques (similar results were observed in other areas). Eleven SIV-infected macaques were imaged with 3D PET using $\left[{ }^{11} \mathrm{C}\right](\mathrm{R})-\mathrm{PK} 11195$ prior to sacrifice, following which they were grouped into mild, moderate, or severe SIVE or SIV-infected, nonencephalitic categories based on the histopathology (frequency of distribution of multinucleated giant cells, microglial nodules, abundant activated and SIV-infected macrophages, perivascular chronic inflammation per $\times 10$ and $\times 40$ field in different brain regions). An increase in $\left[{ }^{11} \mathrm{C}\right](\mathrm{R})$-PK11195-specific binding (represented as percentage of increase in specific binding over controls) corresponded with the postmortem diagnosis of SIVE. Macaques that did not exhibit increased $\left[{ }^{11} \mathrm{C}\right](\mathrm{R})-\mathrm{PK} 11195$ binding did not exhibit SIVE. AMacaque 2263 showed a densely focal but low frequency of distribution of microglial nodules and macrophages and was classified as mild SIVE. BMacaque 9231 had macrophage (staining negative for SIV antigen) infiltration in the basal ganglia (without other histopathological features of SIVE, which might represent a pre-encephalitic state) that may have resulted in the $29 \%$ increase in $\left[{ }^{11} \mathrm{C}\right](\mathrm{R})-\mathrm{PK} 11195$ binding. NA, not assessed.

over 90 minutes in 33 frames. Emission data were corrected for attenuation, dead time, scatter, and radioactive decay.

The $\left[{ }^{11} \mathrm{C}\right](\mathrm{R})-\mathrm{PK} 11195$ PET scans of each macaque were coregistered to the baseline PET scan using the Automated Image Registration algorithm (38). Regions of interest were defined on summed PET images (0-8 minutes after injection) and applied to the dynamic PET images to generate time-activity curves for the midfrontal cortex, basal ganglia, cerebellum, occipital cortex, and hippocampal (mesial temporal lobe) brain regions. In the non-CD8 T cell-depleted animals, the regional brain radioactivity concentration summed over the scan frames at various time points was normalized to both the injected dose of $\left[{ }^{11} \mathrm{C}\right](\mathrm{R})-\mathrm{PK} 11195$ and the body mass of the animal (\% injected dose) to represent a semiquantitative measure of $\left[{ }^{11} \mathrm{C}\right](\mathrm{R})-\mathrm{PK} 11195$ binding.

In the CD8 T cell-depleted macaques, we used the referenceLogan graphical analysis method to determine the specific binding of $\left[{ }^{11} \mathrm{C}\right](\mathrm{R})-\mathrm{PK} 11195$ (39). The $\left[{ }^{11} \mathrm{C}\right](\mathrm{R})-\mathrm{PK} 11195$ PET scans obtained prior to SIV infection allowed each animal to serve as its own control because of the difficulty of identifying a consistent brain region devoid of disease pathology to serve as reference tissue.

MRI. Imaging was performed using a 3.0 Tesla whole-body MRI scanner (General Electric Medical Systems, Milwaukee, Wisconsin,
USA) operating under version VH3 of the scanning software. The scanner has peak gradient strength of $4 \mathrm{G} / \mathrm{cm}$ and peak slew rate of $15,000 \mathrm{G} / \mathrm{cm} / \mathrm{s}$. A custom-built, quadrature, birdcage extremity coil (15 cm diameter, 32 legs) was used for scanning the animals placed supine on the imaging table.

Data acquisition was performed using an imaging protocol of standard imaging sequences used for the evaluation of anatomical detail, followed by the acquisition of a high-resolution gradient echo data set to be used for MRI PET coregistration and a dynamic, contrast-enhanced echo-planar imaging (EPI) acquisition.

High-resolution spoiled gradient-recalled acquisition. Three-dimensional gradient echo images were acquired prior to contrast injection to provide a high-resolution data set for MRI-PET coregistration. This spoiled gradient-recalled data set was acquired using a time to echo, time to repetition, and flip angle combination ( $7 \mathrm{~ms}, 25 \mathrm{~ms}$, and $40^{\circ}$, respectively), chosen to maximize contrast between brain tissue and its surroundings (including meninges and cerebral spinal fluid [CSF]).

Contrast-enhanced gradient echo EPI. Multislice (21 axial slices, 3 $\mathrm{mm}$ thick, no gap, 80 images per slice) gradient-recalled, singleshot, EPI images (flip $=60^{\circ}$ ) were acquired 1 minute before and 1 minute after bolus venous injection $(3 \mathrm{ml}$ saline solution with $0.1 \mathrm{mM} / \mathrm{kg}$ of contrast agent, 1 second) of gadodiamide (Omniscan; Nycomed Inc., Princeton, New Jersey, USA). These data were acquired after collection of all other magnetic resonance data sets. Volume measurements were determined as described before using the MORPH software $(40,41)$.

Autoradiography. Autoradiography was performed using 15$\mu \mathrm{m}$-thick frozen sections obtained from the frontal cortex (29). Brain sections were placed on Superfrost glass slides (Sigma-Aldrich, St. Louis, Missouri, USA) and incubated in ice-cold $50 \mathrm{mM}$ Tris- $\mathrm{HCl}$ ( $\mathrm{pH} 7.4$ ) containing $1 \mathrm{nM}\left[{ }^{3} \mathrm{H}\right](\mathrm{R})$ PK11195 (specific activity, 89.9 Ci/mmol; NEN Life Sciences Products Inc., Boston, Massachusetts, USA) for 30 minutes. Specificity of binding was ensured by the inclusion of $10 \mu \mathrm{M}$ PK11195 (Sigma-Aldrich) in parallel sections. The sections were mounted with a layer of autoradiographic LM-1 emulsion (Amersham International, Amersham, United Kingdom) using a wire loop; they were then developed after 4 weeks and imaged on the confocal microscope.

Filtration radioligand binding assays. Brain tissue from various regions was dissected, weighed, and homogenized in ice-cold $50 \mathrm{mM}$ Tris$\mathrm{HCl}$ buffer ( $\mathrm{pH}$ 7.4). Homogenates were washed four times by centrifugation at $40,000 \mathrm{~g}$ for 20 minutes at $4^{\circ} \mathrm{C}$. Total binding (per milligram of tissue) was determined by incubating tissue (total protein concentrations ranging from 150 to $200 \mu \mathrm{g}$, determined using the BCA protein assay kit; Pierce Chemical Co., Rockford, Illinois, USA) with $1 \mathrm{nM}\left[{ }^{3} \mathrm{H}\right](\mathrm{R})-\mathrm{PK} 11195$, except in saturation binding experiments where $0.5-80 \mathrm{nM}\left[{ }^{3} \mathrm{H}\right](\mathrm{R})-\mathrm{PK} 11195$ was used, at $4^{\circ} \mathrm{C}$ for 2 hours in a final volume of $250 \mu \mathrm{l}$ of Tris- $\mathrm{HCl}$. Nonspecific binding was determined by the inclusion of $10 \mu \mathrm{M}$ PK11195. Specific binding (in femtomoles per milligram of protein incubated in each test tube) was defined as the difference between total and nonspecific binding (29). The reaction was terminated by the addition of ice-cold buffer in a vacuum cell harvester (Brandel Inc., Gaithersburg, Maryland, USA). All samples were run in duplicate.

Immunohistochemical labeling and confocal microscopic quantification. Immunostaining and laser confocal microscopic quantification was performed as described previously $(42,43)$. Sections were 


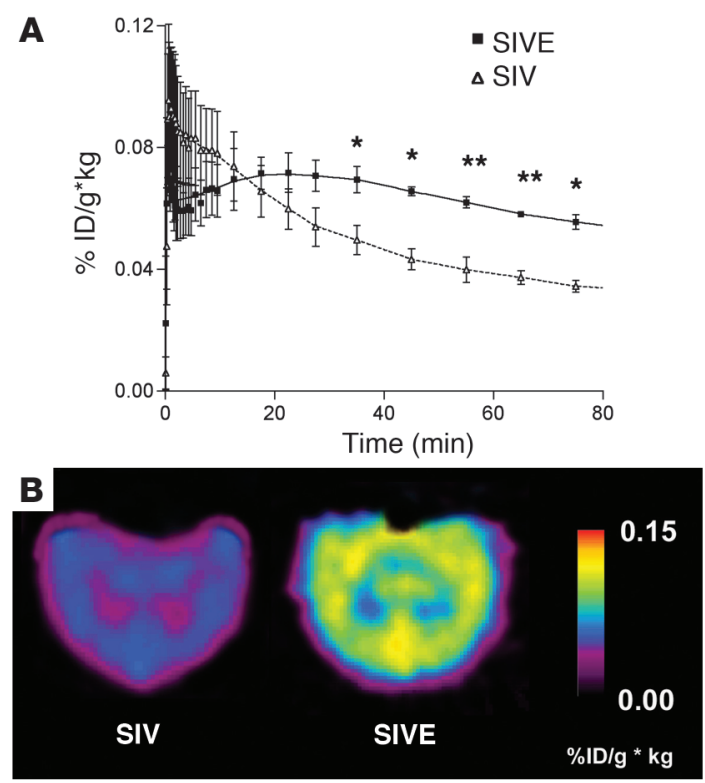

Figure 1

$\left[{ }^{11} \mathrm{C}\right](\mathrm{R})-\mathrm{PK} 11195-$ specific binding is higher in macaques that develop SIVE in vivo compared with SIV-infected macaques that do not develop encephalitis. (A) Time-activity curve of $\left[{ }^{11} \mathrm{C}\right](\mathrm{R})$-PK11195-specific binding in the mesial temporal lobe of non-CD8 $\mathrm{T}$ cell-depleted macaques imaged before necropsy. $\left[{ }^{11} \mathrm{C}\right](\mathrm{R})-\mathrm{PK} 11195-$ specific binding is represented as radioactivity concentrations summed over the scan frames and normalized to both the injected dose of $\left[{ }^{11} \mathrm{C}\right](\mathrm{R})-\mathrm{PK} 11195$ and the body mass of the animal (\% ID $/ \mathrm{g} \times \mathrm{kg}, y$-axis) and is plotted against time in minutes ( $x$ axis). Macaques were then classified into SIVE ( $n=2$, filled squares) and control SIV-infected, nonencephalitic groups (SIV; $n=4$, open triangles) based on histopathology (Table 2). $\left[{ }^{11} \mathrm{C}\right](\mathrm{R})-\mathrm{PK} 11195-$ specific binding determined prior to neuropathological evaluation was compared in these two macaque groups. SIVE macaques show increased $\left[{ }^{11} \mathrm{C}\right](\mathrm{R})-\mathrm{PK} 11195$ retention and specific binding over time in the late scan frames (40-90 minutes) compared with SIV-infected, nonencephalitic macaques. Data was analyzed using the Student's $t$ test with $95 \%$ confidence intervals. ${ }^{* *} P<0.004$, ${ }^{*} P<0.03$. (B) Representative horizontal-section parametric images of $\left[{ }^{11} \mathrm{C}\right](\mathrm{R})$-PK11195-specific binding in SIVE and SIV-infected, nonencephalitic (SIV) macaques. SIVE macaque brains show increased $\left[{ }^{11} \mathrm{C}\right](\mathrm{R})-\mathrm{PK} 11195-$ specific binding compared with SIV-infected, nonencephalitic macaques.

stained with Ab's to glial fibrillary acidic protein (GFAP, mouse mAb; DAKO Corp., Carpinteria, California, USA), or CD68 (mouse mAb; DAKO Corp.), or SIV gp110 (a gift from Kelly Stephano-Cole and Ronald Montelaro, Department of Molecular Genetics and Biochemistry, University of Pittsburgh School of Medicine, Pittsburgh, Pennsylvania, USA), or microtubule-associated protein-2 (MAP-2) (SMI 52; Sternberger Monoclonals Inc., Lutherville, Maryland, USA) used at concentrations 1:500, 1:100, 1:100, and 1:1,000, respectively. Sections were then incubated with cyanine 5-conjugated (Cy5-conjugated) goat anti-mouse or Cy3-conjugated antirabbit IgG at a concentration of 1:200 (Jackson Immunoresearch Laboratories Inc., West Grove, Pennsylvania, USA). Different anatomic regions were identified using a dissection microscope and marked on the slides. Immunostained sections were scanned and quantified on a laser confocal microscope (LSM 150; Carl Zeiss $\mathrm{GmbH}$, Heidelberg, Germany). This scope is equipped with an argon laser with $458 \mathrm{~nm}, 477 \mathrm{~nm}, 488 \mathrm{~nm}$, and $514 \mathrm{~nm}$ primary emission lines. Each section was scanned along the $z$ axis to define the middle optical plane used in quantification $(262,144$ pixels/ plane; 1 pixel $\left.=0.25 \mathrm{~mm}^{2}\right)$. Image analysis was performed on a Silicon Graphics computer (Windows NT 4.0 Operating System; Microsoft Corp., Redmond, Washington, USA) using the LSM software (version 3.0; Carl Zeiss GmbH). Scanning parameters such as laser power aperture, gain, and photomultiplier tube settings were kept constant for each wavelength.

In each anatomic region, an individual blinded to the experimental design imaged ten areas $(\times 40)$ encompassing $106,100 \mu \mathrm{m}^{2}$. For each cell phenotype scanned, contribution to signal intensity from autofluorescence was minimized using a threshold that was kept constant. In each area the average pixel fluorescence along with the pixel counts for a given cell phenotype marker that exceeded the threshold were enumerated. The average pixel fluorescence was multiplied by the total number of pixels to measure the total fluorescence for that cell phenotype marker in that area. The total fluorescence values determined from the ten scanned areas in one brain region were averaged to represent a measure of the cell phenotype in that brain region.

To evaluate cellular localization of $\left[{ }^{3} \mathrm{H}\right](\mathrm{R})-\mathrm{PK} 11195$ binding in SIVE brain tissue we combined immunostaining with autoradiography. Sections were first immunostained, subjected to autoradiography (see above), and then imaged on the confocal microscope.

Statistical analysis. Data were analyzed using PRISM software (GraphPad Software for Science Inc., San Diego, California, USA). Student $t$ tests or one-way ANOVA tests with post-test Bonferroni correction and $95 \%$ confidence intervals were used to analyze data. Correlations using 95\% confidence intervals were performed to quantify the relationship between PK11195 binding and macrophages or astrocytes. Results from correlational analyses are represented by $r$, the Spearman's coefficient.

\section{Results}

$\left[{ }^{11} C\right](R)-P K 11195$ PET binding is higher in macaques that develop SIVE. To test if PET imaging of macaques using $\left[{ }^{11} \mathrm{C}\right](\mathrm{R})$-PK11195 correlates with the development of SIVE, we compared PET findings in SIVDB670-infected macaques immediately prior to sacrifice with neuropathological features. Our macaque cohort consisted of 14 animals (six animals were CD8 T cell depleted to compress the disease time course and eight were non-CD8 T cell depleted) (Table 1). Eleven of these macaques were imaged using 3D PET (Table 1 and 2). Six of the $11 \mathrm{SIV}$-infected macaques (four CD8 T cell-depleted and two non-CD8 $\mathrm{T}$ cell-depleted macaques) demonstrated increased uptake and retention of $\left[{ }^{11} \mathrm{C}\right](\mathrm{R})$-PK11195 with PET compared with the other macaques $(P<0.03$, Table 2 and Figure 1$)$.

Brain histopathology was assessed in all macaques after PET imaging. SIVE was defined by the presence of parenchymal and perivascular macrophages, multinucleated giant cells, microglial nodules, and abundant macrophages that stained for the SIV envelope protein. The six macaques that demonstrated increased $\left[{ }^{11} \mathrm{C}\right](\mathrm{R})-\mathrm{PK} 11195$ PET binding showed various degrees of SIVE (Table 2). Macaque 9231 showed some macrophage infiltration in the basal ganglia without other classical histopathological features of SIVE, which corresponded to a $29 \%$ increase in $\left[{ }^{11} \mathrm{C}\right](\mathrm{R})$ PK11195-specific binding in vivo in the basal ganglia (Table 2) and might represent a pre-encephalitic stage of disease. The remaining macaques either showed subtle inflammatory changes in the form of perivascular mononuclear cell infiltrates or failed to show any 


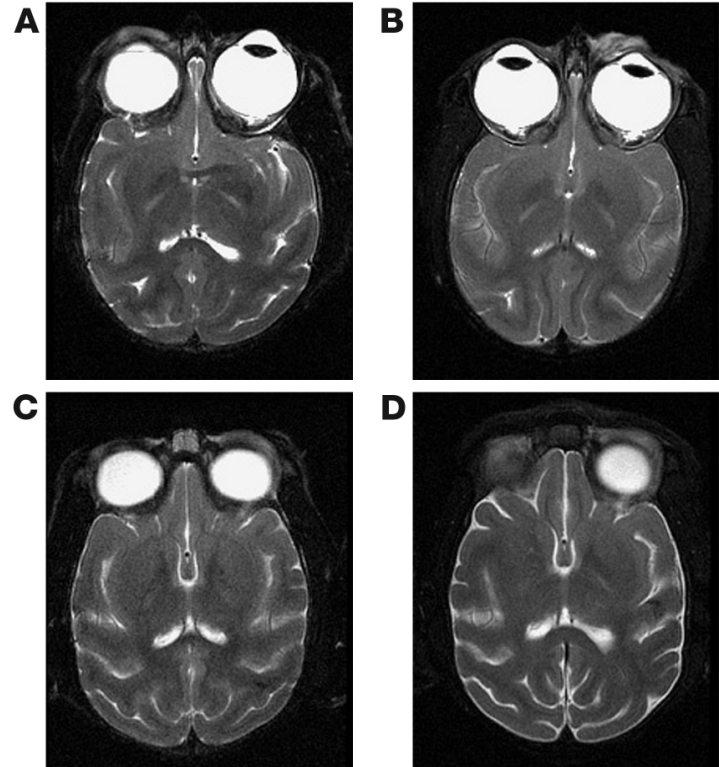

significant abnormalities and were therefore considered nonencephalitic controls. These macaques showed marginal or no PET $\left[{ }^{11} \mathrm{C}\right](\mathrm{R})-\mathrm{PK} 11195$ uptake and binding (Table 2). Increased $\left[{ }^{11} \mathrm{C}\right](\mathrm{R})-$ PK11195-specific binding in vivo correlated with the postmortem diagnosis of SIVE.

MRI imaging of the macaques. Four macaques underwent head MRI with and without contrast administration. Each animal was scanned before infection and then at 1 month after infection and the day before sacrifice. MRI findings were compared with histopathological findings at postmortem examination as described before.

In these macaques, no structural abnormalities were observed in any of the scans at baseline before infection (Figure 2, A and C). No qualitatively appreciable contrast enhancement was observed at any

\section{Figure 2}

Comparison of preinfection and postinfection MRI scans in SIVE macaques. Head MRI scans from two macaques before infection (A and $\mathbf{C}$ ) and immediately before autopsy (right, $\mathbf{B}$ and $\mathbf{D})$. (A and $\mathbf{B}$ ) Images from a macaque that demonstrated mild SIVE at autopsy. No significant changes were observed between preinfection and postinfection images. (C and D) Images from a macaque that demonstrated severe SIVE at autopsy. Mild increase in CSF space and parenchymal atrophy were observed in the macaque. No contrast enhancement was observed in any brain at any time.

time in any animal. At autopsy the macaque without significant neuropathology and the macaque with mild SIVE (Figure 2, A and B) showed no appreciable structural differences between preinfection and postinfection scans with any of the MRI sequences. The macaques with moderate and severe SIVE showed no appreciable structural abnormalities at 1 month after infection. Scans immediately prior to sacrifice, however, showed mild atrophy with ventricular dilatation (average increase in ventricular volume of $47 \%$ relative to preinfection scans, data not shown) best appreciated with T-2 sequences (Figure 2, C and D).

$\left[{ }^{3} H\right](R)-P K 11195$ binding is higher in SIVE brain tissue. To confirm increased PK11195 binding in SIVE macaques we used filtration binding and autoradiographic analyses to compare $\left[{ }^{3} \mathrm{H}\right](\mathrm{R})$ PK11195-specific binding in SIVE brain tissue with controls. $\left[{ }^{3} \mathrm{H}\right](\mathrm{R})-\mathrm{PK} 11195$-specific binding (per milligram of protein) was defined as the difference between total binding (determined by incubation of brain tissue with $\left.1 \mathrm{nM}\left[{ }^{3} \mathrm{H}\right](\mathrm{R})-\mathrm{PK} 11195\right)$ and nonspecific binding (determined by including $10 \mu \mathrm{M}$ PK11195). Specific binding was significantly higher in the frontal white and gray matter, basal ganglia, and hippocampal areas of encephalitic macaques $(P<0.004)$ compared with binding in these regions in SIV-infected, nonencephalitic and noninfected macaque brain tissue (Figure $3 \mathrm{~A}$ and Table 2). The cerebellum and occipital cortex of encephalitic macaques did not show a significant difference in specific binding over these regions in control macaques (Figure

\section{Figure 3}

$\left[{ }^{3} \mathrm{H}\right](\mathrm{R})-\mathrm{PK} 11195-$ specific binding is higher in SIVE brain tissue compared with controls. (A) $\left.{ }^{3} \mathrm{H}\right](\mathrm{R})$-PK11195-specific binding (femtomoles per milligram of protein; $y$ axis) is higher in the frontal cortical white matter (FWM), frontal cortical gray matter (FGM), basal ganglia (BG), and hippocampus (HIP) in SIVE macaques ( $n=3$, black bars) compared with noninfected controls (Con; $n=2$, white bars) and SIV-infected, nonencephalitic (SIV; $n=3$, gray bars) macaques. No significant difference was seen in the cerebellum (CB) and the occipital cortex (OC). Data analyzed using one-sided ANOVA. ${ }^{*} P<0.004$, ${ }^{* *} P<0.0002$. (B) $\left[{ }^{3} \mathrm{H}\right](\mathrm{R})-\mathrm{PK} 11195(0.5-80 \mathrm{nM})$ saturation binding experiments indicate that increase in specific binding is due to an increase in total number of $\left[{ }^{3} \mathrm{H}\right](\mathrm{R})$-PK11195-binding sites $\left(B_{\max }\right.$, femtomoles per milligram of protein, $y$ axis) in SIVE ( $n=3$, black bars) as compared with noninfected controls (Con; $n=2$, white bars) and SIV-infected, nonencephalitic (SIV; $n=3$, hatched bars) frontal cortical brain tissue. Data analyzed using one-sided ANOVA. ${ }^{* *} P=0.009$. (C) Saturation binding experiments indicate no significant increase in $\left[{ }^{3} \mathrm{H}\right](\mathrm{R})-\mathrm{PK} 11195-$ binding affinity $\left(K_{\mathrm{d}}, \mathrm{nM}, y\right.$ axis) in SIVE ( $n=3$, black bars) as compared with noninfected controls (Con; $n=2$, white bars) and SIV-infected, nonencephalitic (SIV; $n=3$, hatched bars) frontal cortical brain tissue. Data analyzed using one-sided ANOVA. $P=0.488$.
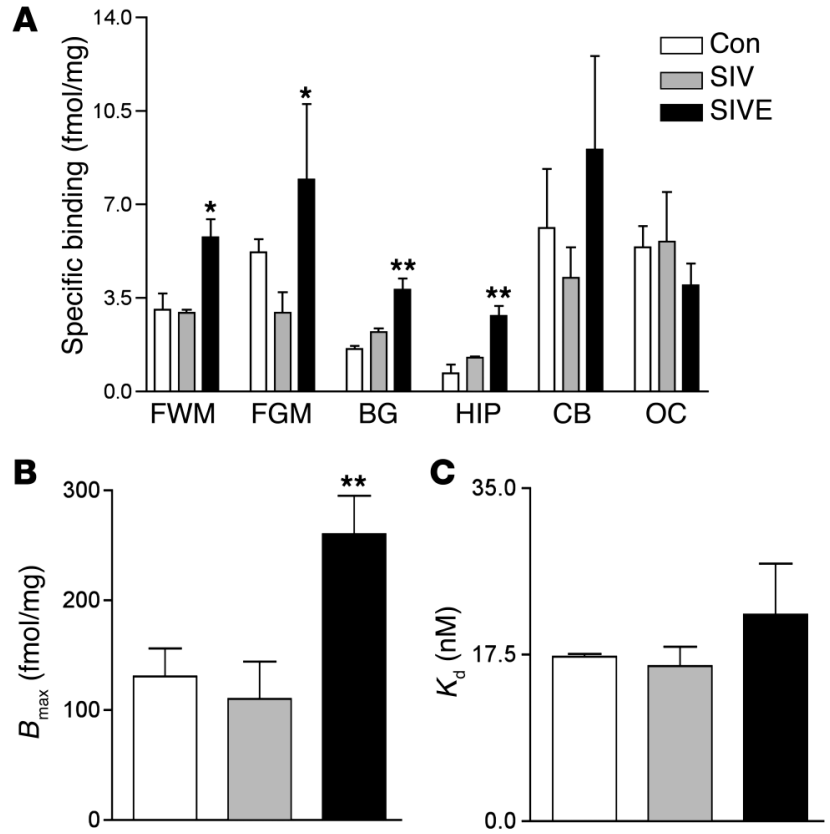

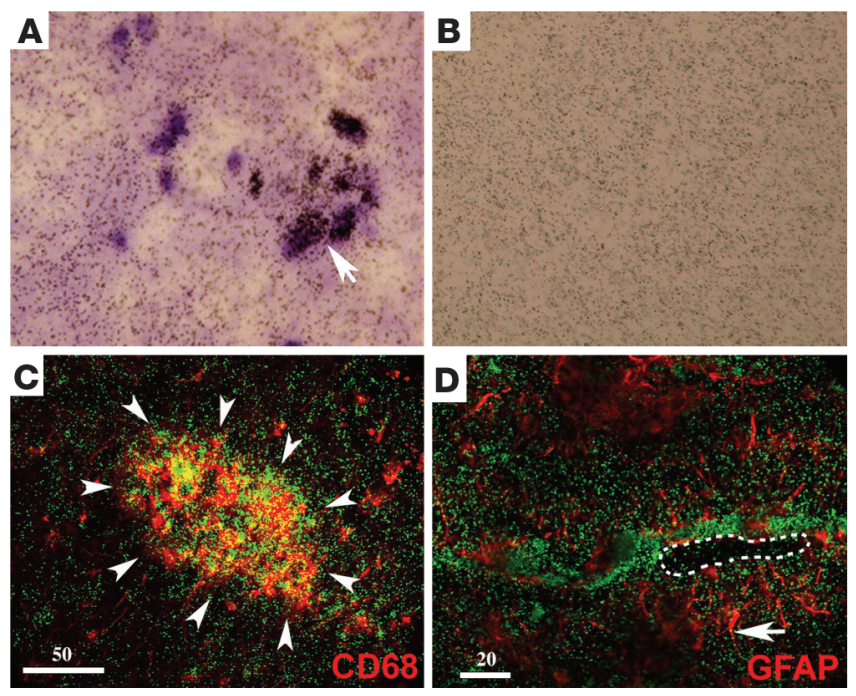

Figure 4

$\left[{ }^{3} \mathrm{H}\right](\mathrm{R})-\mathrm{PK} 11195-$ specific binding in SIVE corresponds to activated macrophages but not astrocytes. (A and $\mathbf{B})\left[{ }^{3} \mathrm{H}\right](\mathrm{R})$-PK11195 autoradiograms (black grains) with light hematoxylin counterstain of sections from frontal cortex show higher specific binding in SIVE (A) compared with SIV-infected, nonencephalitic macaques (B). Arrow in A shows a region of numerous grains overlying a microglial nodule. (C and $\mathbf{D}$ ) Combined $\left[{ }^{3} \mathrm{H}\right](\mathrm{R})$-PK11195 autoradiography (green grains) and immunostaining for activated macrophages (C) (CD68, red) or astrocytes (D) (GFAP, red) in SIVE frontal cortex. [ $\left.{ }^{3} \mathrm{H}\right](\mathrm{R})$-PK11195-specific binding overlaps CD68 (C) and not GFAP (D) immunostaining. Arrowheads in $\mathbf{C}$ surround a microglial nodule. (D) Dashed line showing the lumen of a blood vessel and arrow showing astrocytic end processes (red). Vessels in SIVE are frequently surrounded by mononuclear infiltrates interdigitated between astrocytic processes in regions with abundant $\left[{ }^{3} \mathrm{H}\right](\mathrm{R})-\mathrm{PK} 11195$ grains in green. Scale bar in microns.

3A). For autoradiography, frozen brain sections obtained from the frontal cortex were incubated with $1 \mathrm{nM}\left[{ }^{3} \mathrm{H}\right](\mathrm{R})-\mathrm{PK} 11195$, with nonspecific binding determined by the inclusion of $10 \mu \mathrm{M}$ PK11195. SIVE brain tissue showed higher $\left[{ }^{3} \mathrm{H}\right](\mathrm{R})-\mathrm{PK} 11195$ binding compared with the nonencephalitic control brain tissue (Figure 4, A and B). $\left[{ }^{3} \mathrm{H}\right](\mathrm{R})$-PK11195 binding in encephalitic tissue appeared more intense and corresponded to the distribution of microglial nodules (Figure 4A) in contrast to the diffuse, lessintense binding seen in SIV-infected, nonencephalitic tissue in all observed fields (Figure 4B). Increased $\left[{ }^{3} \mathrm{H}\right](\mathrm{R})$-PK11195-specific binding in postmortem brain tissue also corresponded to an increase in $\left[{ }^{11} \mathrm{C}\right](\mathrm{R})-\mathrm{PK} 11195$ binding in encephalitic macaques in vivo (Table 2 ).

Increased PK11195 binding in SIVE macaques may be due to increases in PBR expression (maximal bound response to ligand $\left[B_{\max }\right]$ per binding sites) or due to alterations in receptor-PK11195 affinity $\left(K_{\mathrm{d}}\right)$. We used $\left[{ }^{3} \mathrm{H}\right](\mathrm{R})-\mathrm{PK} 11195$ saturation-binding experiments (range $0.5-80 \mathrm{nM}$ ) to compare the total number of binding sites $\left(B_{\max }\right)$ and ligand affinity $\left(K_{\mathrm{d}}\right)$ in SIVE with SIV-infected, nonencephalitic, and noninfected controls. SIVE macaques showed a significant increase in $\left[{ }^{3} \mathrm{H}\right](\mathrm{R})$-PK11195-binding sites $\left(B_{\max }\right.$, Figure $3 \mathrm{~B})(P=0.009)$ with no significant difference in ligand affinity $\left(K_{\mathrm{d}} ;\right.$ Figure 3C) $(P=0.488)$.

$\left[{ }^{3} H\right](R)-P K 11195$ binding in SIVE brain tissue localizes to macrophages. PBR is expressed mainly on astrocytes and brain macrophages (27, 44 ) and at lower levels in neurons (45). We wanted to determine the relative contributions of these cell types to $\left[{ }^{3} \mathrm{H}\right](\mathrm{R})-\mathrm{PK} 11195$ binding. We addressed this question in two ways. First, we combined immunostaining for astrocytes (GFAP) and activated macrophages with $\left[{ }^{3} \mathrm{H}\right](\mathrm{R})$-PK11195 autoradiography on frozen brain sections obtained from the frontal cortex of SIVE macaques. (As a macrophage lysosomal marker, CD68 is increased on activation and is useful to label activated macrophages/microglia.) $\left[{ }^{3} \mathrm{H}\right](\mathrm{R})$ PK11195 binding in SIVE overlapped regions rich in activated macrophages and microglial nodules (aggregates of macrophages) (Figure 4C). [ $\left.{ }^{3} \mathrm{H}\right](\mathrm{R})$-PK11195 binding did not overlap GFAPlabeled astrocytes in encephalitic brain tissue (Figure 4D).

Second, we tested whether $\left[{ }^{3} \mathrm{H}\right](\mathrm{R})-\mathrm{PK} 11195$ binding in homogenized brain tissue correlated with the abundance of astrocytes or activated macrophages or neurons. Astrocytes, activated macrophages, and neuronal elements were immunostained for GFAP, CD68, and MAP-2, respectively, and were quantified in SIVE- and SIV-infected, nonencephalitic brain tissue using laser confocal microscopy conducted by an individual blinded to the experimental design. Each cell-type marker was then correlated with $\left[{ }^{3} \mathrm{H}\right](\mathrm{R})-\mathrm{PK} 11195$ filtration-binding values obtained from the corresponding brain regions in the same macaques. Results of correlations are represented by Spearmen's correlation coefficient $r$ (Table 3). $\left[{ }^{3} \mathrm{H}\right](\mathrm{R})$-PK11195 binding correlated with the abundance of CD68-stained activated macrophages $(r=0.5285$; $P=0.0066)$, but not with the abundance of GFAP-stained astrocytes $(r=0.1991$; $P=0.2915)$ or with the abundance of MAP-2-stained neuronal elements $(r=0.0709 ; P=0.6767)$ (Table 3$)$.

\section{Table 3}

$\left[{ }^{3} \mathrm{H}\right](\mathrm{R})-\mathrm{PK} 11195$ binding in postmortem tissue and $\left[{ }^{11} \mathrm{C}\right](\mathrm{R})-\mathrm{PK} 11195$ binding in vivo correlate with immunostaining for activated macrophages but not astrocytes or neurons

\section{Spearmen's coefficient $(r)$ with $\left[{ }^{3} \mathrm{H}\right](\mathrm{R})$-PK11195 binding} in postmortem tissue

$\begin{array}{ccc}\begin{array}{c}\text { Activated macrophages } \\ \text { (CD68) }\end{array} & \begin{array}{c}\text { Astrocytes } \\ \text { (GFAP) }\end{array} & \begin{array}{c}\text { Neurons } \\ \text { (MAP-2) }\end{array} \\ 0.5285^{\mathrm{A}} & 0.1991 & 0.0709\end{array}$

\section{Spearmen's coefficient $(r)$ with [11C](R)-PK11195 binding} in vivo

Activated macrophages
(CD68)
$0.6424^{\mathrm{A}}$

\section{Neurons (MAP-2) \\ 0.0574}

Activated macrophages, astrocytes, and neuronal elements were quantified by immunostaining for CD68 (lysosomal marker for activated macrophages), GFAP (astrocytic marker), and MAP-2 in the frontal cortex, basal ganglia, hippocampus, cerebellum, and occipital cortex of SIVE ( $n=3$ ) and SIV-infected, nonencephalitic $(n=3)$ macaques, using confocal laser microscopy. Each cell phenotype marker was then correlated with [3H](R)-PK11195 binding in postmortem tissue or $\left[{ }^{11} \mathrm{C}\right](\mathrm{R})$-PK11195 PET binding in vivo in the corresponding brain regions in the same macaques. PK11195 binding in postmortem tissue and in vivo correlated with activated macrophages but not astrocytes or neuronal elements, ${ }^{A} P<0.006$. 
$\left[{ }^{11} C\right](R)-P K 11195$ binding in SIVE in vivo correlates with the presence of activated macrophages. We correlated $\left[{ }^{11} \mathrm{C}\right](\mathrm{R})$-PK11195-specific binding in vivo with the presence of astrocytes, activated macrophages, or neuronal elements in selected brain regions of SIVE- and SIV-infected nonencephalitic macaques. Astrocytes, activated macrophages, and neurons were immunostained and quantified using laser confocal microscopy as above and were compared with in vivo estimates of $\left[{ }^{11} \mathrm{C}\right](\mathrm{R})$-PK11195-specific binding in the corresponding brain regions in the same macaques. $\left[{ }^{11} \mathrm{C}\right](\mathrm{R})$ PK11195-binding correlated with the abundance of CD68-stained activated macrophages $(r=0.6424, P=0.0013)$ but did not correlate with the presence of astrocytes $(r=-0.1692, P=0.5630)$ or neurons $(r=0.0574, P=0.563)$ (Table 3$)$.

\section{Discussion}

We examined binding of the PBR ligand PK11195 in a macaque model of HIVE. In vivo ligand binding was assessed using $\left[{ }^{11} \mathrm{C}\right]$ labeled (R)-PK11195 with PET in SIV-infected macaques. Out of 11 imaged SIV-infected macaques, six showed higher levels of $\left[{ }^{11} \mathrm{C}\right](\mathrm{R})$ PK11195 binding (Figure 1 and Table 2). Increased binding corresponded to the presence of SIVE on postmortem examination (Table 2). Macaques that did not show an increase in $\left[{ }^{11} \mathrm{C}\right](\mathrm{R})$ PK11195 binding did not exhibit SIVE (Table 2). In four macaques comparison of MRI before infection and 2 months after infection showed mild brain atrophy and increase in ventricular size only in the moderate to severe SIVE cases (Figure 2 and data not shown). Brain postmortem $\left[{ }^{3} \mathrm{H}\right](\mathrm{R})-\mathrm{PK} 11195$ binding was significantly higher in SIVE macaques from controls due to an increase in the number of binding sites $\left(B_{\max }\right)$ with no significant changes in binding affinity $\left(K_{\mathrm{d}}\right)$ (Figure 3 ) and correlated with in vivo binding measured with PET just before sacrifice (Table 2 and data not shown). Brain regions with elevated PK11195 binding on PET and filtration assays correlated with abundant activated macrophages but not with activated astrocytes or neurons quantified by laser confocal microscopy (Table 3). Finally, combined autoradiography and immunostaining identified macrophages, but not astrocytes, as the cells with highest $\left[{ }^{3} \mathrm{H}\right](\mathrm{R})$-PK11195 binding (Figure 4).

Our study involves the use of two different species of macaques and uses two different protocols (with and without CD8 T cell depletion) to induce disease. While this is a limitation based on macaque availability and continual evolution of the primate model, baseline preinfection PET studies in animals of the two different species did not indicate significant differences (average PET $\left[{ }^{11} \mathrm{C}\right](\mathrm{R})$-PK11195 binding in the basal ganglia of rhesus macaques, $0.06 \% \pm 0.01 \%$ injected dose of PK11195 per grams of brain tissue $\times$ body weight of animal $(\mathrm{ID} / \mathrm{g} \times \mathrm{kg})$, versus pigtail macaques, $0.08 \% \pm 0.02 \% \mathrm{ID} / \mathrm{g} \times \mathrm{kg}$, $P=0.217$ ). Similarly, other brain regions, including the frontal cortex, cerebellum, occipital cortex, and hippocampus, showed no significant differences between the two species. Thus, data from all control animals were grouped together. We cannot rule out the possibility of differences resulting from species in the extent of CNS disease, however. Furthermore, it has been suggested by Williams and Hickey (46) that the CD8 $\mathrm{T}$ cell depletion results in an increase in the severity of CNS disease, and this was the impetus to our using this model. Preliminary histopathological comparisons of our two groups of animals did not indicate any major differences (Table 2). We are unable to draw any conclusions regarding this issue, however, due to the small number of CD8 T cell-depleted macaques used in this study.

In the frontal cortex, mesial temporal lobe, and the basal ganglia of SIVE macaques, $\left[{ }^{3} \mathrm{H}\right](\mathrm{R})$-PK11195 binding in postmortem tissue correlated with PET $\left[{ }^{11} \mathrm{C}\right](\mathrm{R})$-PK11195 binding in vivo (Table 2 and data not shown). In the cerebellum and occipital cortex PET $\left[{ }^{11} \mathrm{C}\right](\mathrm{R})-\mathrm{PK} 11195$ binding showed significant increases that we did not see in postmortem tissue (Figure 1 and Figure 3 and data not shown). Furthermore, while macaque 2263 showed $213 \%$ increase in PET binding from control in the basal ganglia, it demonstrated mild but densely focal encephalitis on postmortem examination in this region. These discrepancies may be due to differences in resolution and sampling size between PET, postmortem tissue-filtration binding analyses, and histopathological assessment. Neuropathological studies are performed on a section of a given brain region and postmortem binding analysis is restricted to a small mass of brain tissue $(\sim 100 \mathrm{mg})$ in contrast to larger regions of the brain involved in PET analyses with more limited spatial resolution. In both postmortem tissue and in vivo PET, however, PK11195 binding closely correlated with the histopathological abundance of macrophages (Table 3 and Figure 4).

Comparing preinfection MRI scans with scans at autopsy in four infected macaques showed mild atrophy with ventricular dilatation only in macaques with moderate and severe SIVE. The macaque with mild SIVE did not show any structural changes on comparing preinfection and postinfection scans but showed regional increases in in vivo PK11195 binding corresponding with macrophage infiltration. This suggests that PET $\left[{ }^{11} \mathrm{C}\right](\mathrm{R})$-PK11195 imaging may be more sensitive than structural MRI in assessing neurologic disease in SIVE, although all of the 11 PET-scanned macaques were not imaged using MRI.

The distribution of lesions in SIVE is multifocal without a prominent or consistent neuroanatomical distribution (42). This diffuse and multifocal distribution hinders identification of a consistent reference region for application of PET reference tissue-based analyses. To overcome this issue, the segmentation of dynamic PET images into clusters of image voxels with similar concentration time-activity curves has been proposed as a viable means to extract a reference ligand kinetic without a priori knowledge of the brain distribution of PBR sites (47). This reference kinetic, though not necessarily derived from a uniform brain region, has been demonstrated to be capable of providing a reasonable estimate of free and nonspecific binding and has been successfully employed in the study of multiple sclerosis patients with $\left[{ }^{11} \mathrm{C}\right](\mathrm{R})$-PK11195 (27).

Although all macaques with increased $\left[{ }^{11} \mathrm{C}\right](\mathrm{R})$-PK11195 binding showed encephalitis, the percentage of increase in $\left[{ }^{11} \mathrm{C}\right](\mathrm{R})$-PK11195 binding from controls was higher in CD8 T cell-depleted SIVE macaques (174-313\%) vis-à-vis the non-CD8 $\mathrm{T}$ cell-depleted macaques (39-55\%) (Table 2). These two groups of encephalitic macaques did not show significant neuropathological differences (Table 2). We attribute this discrepancy to the incommensurability of the two parameters used to estimate specific binding. In the non-CD8 T cell-depleted animals, a semiquantitative measure of $\left[{ }^{11} \mathrm{C}\right](\mathrm{R})-\mathrm{PK} 11195$ binding was defined to be the regional brain radioactivity concentration summed over the late scan frames (Figure 1, 40-90 minutes after injection) and normalized to both the injected dose of $\left[{ }^{11} \mathrm{C}\right](\mathrm{R})-\mathrm{PK} 11195$ and the body mass of the animal $(\% \mathrm{ID} / \mathrm{g} \times \mathrm{kg})$. In contrast, $\left[{ }^{11} \mathrm{C}\right](\mathrm{R})$-PK11195-specific binding in the CD8 T cell-depleted animals was determined using each macaque's regional baseline (before infection) $\left[{ }^{11} \mathrm{C}\right](\mathrm{R})-\mathrm{PK} 11195$ time-activity curve as the reference kinetic for regional postinfection assessments based on the reference-Logan graphical analysis method $(27,48,49)$. Because the reference tissue kinetic provides an estimate of both free and nonspecifically bound radiotracer, the binding potential 
parameter derived by this method of analysis from the CD8 T cell-depleted macaques represents a more accurate measure of specific binding. This is in contrast to the more qualitative estimates of specific binding (regional brain radioactivity concentrations without subtraction of the free and nonspecific binding) obtained over the summed late PET scan frames from the first group of non-CD8 $\mathrm{T}$ cell-depleted macaques.

We also found that in the CD8 T cell-depleted group, normalized regional brain radioactivity concentrations $(\% \mathrm{ID} / \mathrm{g} \times \mathrm{kg})$ were considerably more variable than in non-CD8-depleted animals (data not shown). Such a discrepancy could be explained by differences in systemic infection with variation in non-CNS macrophage proliferation and subsequent specific binding of $\left[{ }^{11} \mathrm{C}\right](\mathrm{R})-\mathrm{PK} 11195$ outside the CNS. Such sites of systemic binding of $\left[{ }^{11} \mathrm{C}\right](\mathrm{R})$ PK11195 could be expected to decrease the partitioning of the ligand in the brain with a varying proportion of the injected dose being taken up into these non-CNS sites of systemic infection.

A recent report by Lockhart et al. (50) has shown that the concentration of PBR sites in the whole blood of healthy human volunteers to be on the order of $10 \mathrm{nM}$, with approximately $85 \%$ of the ligand bound in plasma and $15 \%$ bound to blood cells. These investigators demonstrated that $\left[{ }^{11} \mathrm{C}\right](\mathrm{R})$-PK11195 binds with high affinity to $\alpha 1$-acid glycoprotein (AGP), which is present in varying degrees in serum. It is possible that binding of $\left[{ }^{11} \mathrm{C}\right](\mathrm{R})-\mathrm{PK} 11195$ to AGP is higher with CD8 T cell depletion in SIV infection, contributing to the unstable kinetic behavior of $\left[{ }^{11} \mathrm{C}\right](\mathrm{R})-\mathrm{PK} 11195$ in plasma. Future studies defining an arterial input function for the injected PK11195 might clarify these modeling problems.

Our results in SIVE are in accordance with previous reports where PK11195 binding (as determined using postmortem tissue and PET) has been used as a marker for brain macrophages and a surrogate marker for neuronal damage $(25,51)$. To determine if PK11195 binding correlated better with SIV-infected macrophages, we correlated PK11195 binding with SIV antigen gp110 quantified using immunostaining in selected brain regions of SIV-infected macaques. The abundance of the viral antigen varied in different regions of the brain, consistent with the multifocal nature of the disease $(42,52)$. Regions with high viral antigen showed high PK11195 binding, but high PK11195 binding was also seen in regions that did not show high viral antigen (data not shown), thus correlating better with activated macrophages. This is presumably due to the more general distribution of activated macrophages in SIVE than viral infection, which is more multifocal $(42,52)$. Our findings support the hypothesis that PK11195 binding may be a surrogate marker of activated macrophages and active loci of neurodegenerative disease in lentivirus encephalitis.

Our results in SIV-infected macaques show that PET assessment of $\left[{ }^{11} \mathrm{C}\right](\mathrm{R})-\mathrm{PK} 11195$ binding can distinguish macaques with and without SIVE (defined at postmortem analysis) in vivo. We are currently conducting serial PET experiments in SIV-infected macaques to determine the earliest time period where this dis- tinction can be made. Importantly, our macaque data can be extended to PET studies in HIV-infected humans. One in four late-stage AIDS patients is afflicted by HIVE in the absence of opportunistic infections (2). Its clinical diagnosis requires the presence of irreversible clinical symptoms and signs. Traditional imaging studies such as computed tomography and MRI have not been of much assistance in the diagnosis of AIDS dementia and dementia in general (53). Current methodologies, such as structural MRI assessment of brain volume, are insensitive late measures of neurological damage, and the diagnosis by neurocognitive tests is possible only after irreversible neuronal damage has occurred. Effective therapy requires early intervention at the onset of neuronal injury prior to the appearance of irreversible signs and symptoms. PET imaging of macrophages in HIVE before the onset of signs and symptoms may enable early diagnosis and therapeutic interventions.

To design therapies for neurodegenerative disorders, it is critical to be able to monitor their success in arresting the progression of neurological disease. Monitoring therapeutic efficacy of dementia therapies is complicated by the fact that one can only assess absence of disease progression and not recovery of function. Any attempt to develop therapy for AIDS dementia will require some means of monitoring the primary pathogenic process: activated macrophages. Issues of CNS drug penetrance and the absence of good markers of treatment effectiveness complicate monitoring the course of HIVE. Imaging macrophages may provide a better index of disease progression during treatment with antiretroviral and other potentially neuroprotective drugs. $\left[{ }^{11} \mathrm{C}\right](\mathrm{R})-\mathrm{PK} 11195$ PET imaging of macrophages may be able to help in the early prediction of HIVE, monitor the severity and progression of the disease, and help evaluate the effectiveness of CNS therapies.

\section{Acknowledgments}

We thank Jonette Werley for valuable technical assistance; Gary Doheny for the MORPH analysis; William E. Klunk, Manik Debnath, Nicole Velez, and Lauren Makuch for help with the binding assays; Rafael Medina-Flores for helpful comments; and Dawn L. ClementsMcBride for assistance in obtaining clinical information about the macaques. CD8 T cell-specific Ab (cM-T807) was produced by the National Cell Culture Center with funds provided by NIH grant RR16001. This work was supported by NIH grant RO1 MH-64921 and K24 MH-01717 (to C.A. Wiley) and the Elizabeth Glaser Pediatric AIDS foundation short-term award 5701964 (S. Venneti).

Received for publication October 7, 2003, and accepted in revised form January 20, 2004.

Address correspondence to: Clayton A. Wiley, Presbyterian University Hospital, Neuropathology Division, 200 Lothrop Street A515, Pittsburgh, Pennsylvania 15213, USA. Phone: (412) 647-0765; Fax: (412) 647-5602; E-mail: wiley@np.awing.upmc.edu.
1. Dore, G.J., et al. 1999. Changes to AIDS dementia complex in the era of highly active antiretroviral therapy. AIDS. 13:1249-1253.

2. Brew, B.J. 1999. AIDS dementia complex. Neurol. Clin. 17:861-881.

3. Cinque, P., Scarpellini, P., Vago, L., Linde, A., and Lazzarin, A. 1997. Diagnosis of central nervous system complications in HIV-infected patients: cerebrospinal fluid analysis by the polymerase chain reaction. AIDS. 11:1-17.

4. McArthur, J.C. 1997. NeuroAIDS: diagnosis and management. Hosp. Prac. (Off. Ed.) 32:73-74, 77-79, 84, passim.

5. Price, R.W., et al. 1999. Neurological outcomes in late HIV infection: adverse impact of neurological impairment on survival and protective effect of antiviral therapy. AIDS Clinical Trial Group and Neurological AIDS Research Consortium study team. AIDS. 13:1677-1685.

6. Baskin, G.B., Murphey-Corb, M., Roberts, E.D., Didier, P.J., and Martin, L.N. 1992. Correlates of SIV encephalitis in rhesus monkeys. J. Med. Primatol.
21:59-63

7. Budka, H. 1991. Neuropathology of human immunodeficiency virus infection. Brain Pathol. 1:163-175.

8. Lackner, A.A., et al. 1991. Localization of simian immunodeficiency virus in the central nervous system of rhesus monkeys. Am. J. Pathol. 139:609-621.

9. Kaul, M., Garden, G.A., and Lipton, S.A. 2001. Pathways to neuronal injury and apoptosis in HIV-associated dementia. Nature. 410:988-994.

10. Post, M.J., et al. 1988. CT, MR, and pathology in 
HIV encephalitis and meningitis. AJR Am.J. Roentgenol. 151:373-380.

11. Avison, M.J., Nath, A., and Berger, J.R. 2002. Understanding pathogenesis and treatment of HIV dementia: a role for magnetic resonance? Trends Neurosci. 25:468-473.

12. Paul, R., Cohen, R., Navia, B., and Tashima, K. 2002. Relationships between cognition and structural neuroimaging findings in adults with human immunodeficiency virus type-1. Neurosci. Biobehav. Rev. 26:353-359.

13. McArthur, J.C., et al. 1990. Incidental white matter hyperintensities on magnetic resonance imaging in HIV-1 infection. Multicenter AIDS Cohort Study. J. Acquir. Immune Defic. Syndr. 3:252-259.

14. Sonnerborg, A., et al. 1990. Quantitative detection of brain aberrations in human immunodeficiency virus type 1 -infected individuals by magnetic resonance imaging. J. Infect. Dis. 162:1245-1251.

15. Post, M.J., et al. 1992. Sequential cranial MR findings of asymptomatic and neurologically symptomatic HIV+ subjects. AJNR Am. J. Neuroradiol. 13:359-370.

16. Raininko, R., et al. 1992. Radiological study of the brain at various stages of human immunodeficiency virus infection: early development of brain atrophy. Neuroradiology. 34:190-196.

17. Aylward, E.H., et al. 1993. Reduced basal ganglia volume in HIV-1-associated dementia: results from quantitative neuroimaging. Neurology. 43:2099-2104.

18. Wenserski, F., von Giesen, H.J., Wittsack, H.J., Aulich, A., and Arendt, G. 2003. Human immunodeficiency virus 1 -associated minor motor disorders: perfusion-weighted MR imaging and $\mathrm{H}$ MR spectroscopy. Radiology. 228:185-192.

19. Pascal, S., et al. 1991. Metabolic asymmetries in asymptomatic HIV-1 seropositive subjects: relationship to disease onset and MRI findings. J. Nucl. Med. 32:1725-1729.

20. Rosci, M.A., et al. 1992. Methods for detecting early signs of AIDS dementia complex in asymptomatic HIV-1-infected subjects. AIDS. 6:1309-1316.

21. Chang, L., Ernst, T., Leonido-Yee, M., Walot, I., and Singer, E. 1999. Cerebral metabolite abnormalities correlate with clinical severity of HIV-1 cognitive motor complex. Neurology. 52:100-108.

22. Chang, L., et al. 2001. Neural correlates of attention and working memory deficits in HIV patients. Newrology. 57:1001-1007.

23. Ernst, T., Chang, L., and Arnold, S. 2003. Increased glial metabolites predict increased working memory network activation in HIV brain injury. Neuroimage. 19:1686-1693.

24. Banati, R.B. 2002. Visualising microglial activation in vivo. Glia. 40:206-217.

25. Cagnin, A., Gerhard, A., and Banati, R.B. 2002. In vivo imaging of neuroinflammation. Eur. Neuropsychopharmacol. 12:581-586.

26. Vowinckel, E., et al. 1997. PK11195 binding to the peripheral benzodiazepine receptor as a marker of microglia activation in multiple sclerosis and experimental autoimmune encephalomyelitis. J. Neurosci. Res. 50:345-353.

27. Banati, R.B., et al. 2000. The peripheral benzodiazepine binding site in the brain in multiple sclerosis: quantitative in vivo imaging of microglia as a measure of disease activity. Brain. 123:2321-2337.

28. Stephenson, D.T., et al. 1995. Peripheral benzodiazepine receptors are colocalized with activated microglia following transient global forebrain ischemia in the rat. J. Neurosci. 15:5263-5274.

29. Raghavendra Rao, V.L., Dogan, A., Bowen, K.K., and Dempsey, R.J. 2000. Traumatic brain injury leads to increased expression of peripheral-type benzodiazepine receptors, neuronal death, and activation of astrocytes and microglia in rat thalamus. Exp. Neurol. 161:102-114.

30. Debruyne, J.C., et al. 2003. PET visualization of microglia in multiple sclerosis patients using [11C]PK11195. Eur. J. Neurol. 10:257-264.

31. Banati, R.B., et al. 1999. [11C](R)-PK11195 positron emission tomography imaging of activated microglia in vivo in Rasmussen's encephalitis. Newrology. 53:2199-2203.

32. Cagnin, A., et al. 2001. In vivo visualization of activated glia by [11C](R)-PK11195-PET following herpes encephalitis reveals projected neuronal damage beyond the primary focal lesion. Brain. 124:2014-2027.

33. Cagnin, A., et al. 2001. In-vivo measurement of activated microglia in dementia. Lancet. 358:461-467.

34. Gerhard, A., et al. 2003. [(11)C](R)-PK11195 PET imaging of microglial activation in multiple system atrophy. Neurology. 61:686-689.

35. Schmitz, J.E., et al. 1999. Control of viremia in simian immunodeficiency virus infection by CD8+ lymphocytes. Science. 283:857-860.

36. Shah, F., Hume, S.P., Pike, V.W., Ashworth, S., and McDermott, J. 1994. Synthesis of the enantiomers of [N-methyl-11C]PK 11195 and comparison of their behaviours as radioligands for PK binding sites in rats. Nucl. Med. Biol. 21:573-581.

37. Cleij, M.C., Aigbirhio, F.I., Baron, J.C., and Clark, J.C. 2003. Base-promoted dechlorination of (R)[C-11]PK-11195. International Symposium on Radiopharmacentical Chemistry, 15th. Sydney, Australia. 46(Suppl.1):588.

38. Woods, R.P., Cherry, S.R., and Mazziotta, J.C. 1992. Rapid automated algorithm for aligning and reslicing PET images. J. Comput. Assist. Tomogr. 16:620-633.

39. Logan, J., et al. 1996. Distribution volume ratios without blood sampling from graphical analysis of
PET data. J. Cereb. Blood Flow Metab. 16:834-840. 40. Johnson, L.A., Pearlman, J.D., Miller, C.A., Young, T.I., and Thulborn, K.R. 1993. MR quantification of cerebral ventricular volume using a semiautomated algorithm. AJNR Am. J. Neuroradiol. 14:1373-1378.

41. Joe, B.N., et al. 1999. Brain tumor volume measurement: comparison of manual and semiautomated methods. Radiology. 212:811-816.

42. Bissel, S.J., et al. 2002. Macrophages relate presynaptic and postsynaptic damage in simian immunodeficiency virus encephalitis. Am. J. Pathol. 160:927-941.

43. Jordan-Sciutto, K.L., Wang, G., Murphey-Corb, M., and Wiley, C.A. 2002. Cell cycle proteins exhibit altered expression patterns in lentiviral-associated encephalitis. J. Neurosci. 22:2185-2195.

44. Casellas, P., Galiegue, S., and Basile, A.S. 2002. Peripheral benzodiazepine receptors and mitochondrial function. Neurochem. Int. 40:475-486.

45. Jayakumar, A.R., Panickar, K.S., and Norenberg, M.D. 2002. Effects on free radical generation by ligands of the peripheral benzodiazepine receptor in cultured neural cells. J. Neurochem. 83:1226-1234.

46. Williams, K.C., and Hickey, W.F. 2002. Central nervous system damage, monocytes and macrophages, and neurological disorders in AIDS. Annu. Rev. Neurosci. 25:537-562.

47. Gunn, R.N., et al. 1998. Tracer kinetic modeling of the 5-HT1A receptor ligand [carbonyl-11C]WAY100635 for PET. Neuroimage. 8:426-440.

48. Lammertsma, A.A., and Hume, S.P. 1996. Simplified reference tissue model for PET receptor studies. Neuroimage. 4:153-158.

49. Gunn, R.N., Lammertsma, A.A., Hume, S.P., and Cunningham, V.J. 1997. Parametric imaging of ligand-receptor binding in PET using a simplified reference region model. Neuroimage. 6:279-287.

50. Lockhart, A., et al 2003. The peripheral benzodiazepine receptor ligand PK11195 binds with high affinity to the acute phase reactant $\alpha 1$-acid glycoprotein: implications for the use of the ligand as a CNS inflammatory marker(1). Nucl. Med. Biol. 30:199-206.

51. Mankowski, J.L., Queen, S.E., Tarwater, P.J., Adams, R.J., and Guilarte, T.R. 2003. Elevated peripheral benzodiazepine receptor expression in simian immunodeficiency virus encephalitis. J. Neurovirol. 9:94-100.

52. Glass, J.D., Fedor, H., Wesselingh, S.L., and McArthur, J.C. 1995. Immunocytochemical quantitation of human immunodeficiency virus in the brain: correlations with dementia. Ann. Neurol. 38:755-762.

53. Jack, C.R., Jr., et al. 2003. MRI as a biomarker of disease progression in a therapeutic trial of milameline for AD. Neurology. 60:253-260. 